\title{
Au-delà de l'épistémologie : le questionnement interminable des sciences sociales
}

In: Quaderni. N. 27, Automne 1995. pp. 53-58.

Citer ce document / Cite this document :

Chanial Philippe. Au-delà de l'épistémologie : le questionnement interminable des sciences sociales. In: Quaderni. N. 27, Automne 1995. pp. 53-58.

doi : 10.3406/quad.1995.1121

http://www.persee.fr/web/revues/home/prescript/article/quad_0987-1381_1995_num_27_1_1121 


\section{Théorie}

\section{AU-DELĀ DE L'ÉPISTÉMOLOGIE : LE QUESTIONNEMENT INTERMINABLE DES SCIENCES SOCIALES}

PHILIPPE CHANIAL

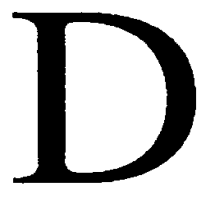

ans leur quête de reconnaissance, les sciences sociales se sont efforcées de revendiquer et d'asseoir lcur légitimité par l'argument épistémologique et ses impératifs de rupture. Distinguant entre fait et valcur, écartant tout ce qui ne relève pas de la connaissance, elles ont prétendu ainsi atteindre à une pure description de la réalité telle qu'elle est, de sorte que l'on puisse en dégager des règles générales. Opposant radicalement le discours scientifique au savoir de sens commun, elles ont forgé un langage explicatif autonome, dissocié de ces discours par lesquels les acteurs rendent comptent eux-mêmes de ce qu'ils sont, de ce qu'ils font. Par cette double rupture, l'éthique ne parait guère trouver sa place au sein des sciences sociales. Elle y resurgit alors simplement comme objet, un objet d'investigation comme un autre, soit comme problème, celui de l'utilisation ou des applications que l'on fait de ses résultats et de ses méthodes.

Note critique sur G.Gosselin, Une Éthique des Sciences Sociales, L'Harmattan, 1992.

Maître de Conférences LASAR - Université de Caen
Tout l'intérêt de l'ouvrage de G.Gosselin est de proposer de réinscrire le questionnement éthique au cœur même des Sciences sociales, sans s'attarder sur d'interminables querelles épistémologiques ni se lamenter sur les usages et mésusages, récupérations et déformations profanes de la recherche scientifique. Le projet de l'auteur est d'emblée énoncé : "Le vrai pou- 
voir du scientifique n'est pas sur les usages qu'il crée : il est dans la création même. C'est donc là, au cour de la démarche, qu'il faut porter l'injonction éthique". Questionner l'éthique de la recherche, c'cst alors expliciter ses postulats, rappeler que l'interrogation des sciences sociales, au-delà de la technicité de ses méthodes et les raffinements de ses constructions épistémologiques, est indissociable d'une anthropologie normative. S'opère ainsi un déplacement du point de vue orthodoxe, le point de vue critique, celui qui tente de débusquer systématiquement - pour les dénoncer - toutes orientations normatives (ou ce que l'on appelait il y a peu "idćologie"). Cette méthode n'est pas tant déniće que questionnéc sur ses conditions de possibilité. Or à moins d'adopter un ethos scientifique totalement désincarné, de faire sien l'idćal d'une "version scientifique de la 'caméra cachée"', de chercher à "capter les croyances et les pratiques comme des entomologistes", il faut rappeler que la posture critique ne saurait exister dans un vide moral, qu'elle présuppose une appartenance à une "communauté de recherche", ellemême situce dans des institutions et des traditions spécifiques.

En ce sens, G.Gosselin, à la suite des chercheurs de l'un de ses ouvragse de référen$c c(1)$, rappelle que toute recherche est $p u$ - blique, que son horizon est moins l'observation désengagćc, la vérification arithmétique, la compilation statistique que le débat, où le chercheur doit s'appliquer à clarifier tant les présupposés de ceux qu'il questionne lors de ses enquêtes que ses propres préconceptions. L'exigence d'auto-réflexion n'est alors que la condition d'une réappropriation de la science par ceux qui la font autant que par ccux pour qui clle est faite. Elle s'impose comme l'ultime butée contre une science devenue autonome, "devenue une puissance qui ne roule plus pour personne, mais sur clle-même".

Le modèle d'une tclle éthique discursive des sciences sociales, l'autcur le découvre dans la recherche biomédicale contemporaine. Qu'elle ait provoqué les discussions que l'on sait - ct l'invention puis l'institutionnalisation de la "bioćthique" - , cela n'exprime-t-il pas, de façon symptomatique, qu'il n'y a plus aujourd'hui de légitimité évidente pour la science, ses découvertes et son pouvoir? C'est donc dans ce contexte plus général d'une crise des modèles transcendants - naturalistes ou scientistes - que tout science, la biologie comme l'anthropologic, doit questionner son éthique implicite afin de reconstruire, sur un autre fondement que l'idéal de puissance et de domination, un modèle de légiti- 
mité alternatif, répondant aux exigences qui lui adresse cette "société post-séculière" que nous décril l'auteur. Qu'il s'agisse du manifeste autour de Serge Leclaire proposant la constitution d'une instance ordinale pour la psychanalyse, de la polémique des historiens allemands sur l'interprétation du nazisme, de la controverse relativisme/universalisme en anthropologie ou enfin du débat en sociologie sur les apports et les apories de la probabilité statistique, G.Gosselin se propose de relire ces quelques débats contemporains en sciences sociales en y dévoilant systématiquement l'urgence de cette injonction éthique, affirmée ou refoulcee.

Les deux derniers chapitres concluant l'ouvrage démontrent que ce parcours, parfois rapide, n'a pas été vain. Il lui permet de formuler et de systématiser les contours et les principes d'une éthique de la recherche en Sciences Sociales. Trois postulats en structurent le projet : la reconnaissance en tout autre d'un autre sujet, d'un alter ego; l'affirmation d'une unité de l'homme, d'une commune humanité ; le primat de l'autonomie de la personne et du rôle structurant de l'intersubjectivité humaine contre toute hypostase des entités collectives, de l'ordre social. L'éthique retrouve alors, au final, l'ćpistémologie, dans l'espace un peu incertain, tant les ré- férences abondent, d'une "socio-anthropologie interprétative" qui vient retrouver les postulats de l'individualisme méthodologique, que l'auteur attribue, sans discussion, à la sociologie weberienne.

C'est autour de cette articulation entre épistémologie et éthique que le lectcur est tenté d'introduire quelques remarques critiques. En effet, autant le questionnement éthique de l'auteur est stimulant autant ses conclusions d'ordre épistémologique sont parfois décevantes. L'individualisme méthodologique prôné par l'autcur, même débarrassé de ses scories rationalistes ou utilitaristes, même corrigé par une inscription de ce paradigme dans l'horizon phénoménologique de l'intersubjectivité, nous offre-t-il véritablement les outils nécessaires à la saisie du sens - éthique - propre à l'action humaine ? Rapporter ultimement la normativité à la subjectivité, à une conscience singulière qui scule pose l'acte éthique, n'est-ce pas tenter, une nouvelle fois, de reconduire subrepticement l'antinomie du sujet et de l'objet, donc de situer cette normativité à l'extérieur de la connaissance scientifique ? Réinjecter de l'éthique dans la science, soumettre l'hubris scientifique à l'injonction éthique du sujet, n'est-ce pas présupposer malgré tout que se font face deux jeux de langage incommensurables que seul un acte volonta- 
riste pourrait enfin réunir?

S'il s'agit bien d'effriter l'antinomie de la vérité et de la normativité en science sociale, deux autres stratégies, participant de ce même projct, peuvent être suggérées, moins pour invalider que pour approfondir la réflexion menće par l'autcur. La première consiste à dénoncer "l'oubli du politique" (2) dans les sciences sociales contemporaines, mais pour rappeler immédiatement que celles-ci, avant de se constituer en disciplines ou sous-disciplines spécialisées, licux où des spécialistes pourraicnt s'appropricr un savoir dernier, n'ont été historiquement que l'une des expressions de ce questionnement certes réaliste et descriptif mais autant utopique, normatif et prescriptif - de la Modernité sur elle-même, de ce moment d'auto-réflexion collective des socićtés, interrogeant autant ce qu'elles sont que ce qu'elles doivent ĉtre. Et de conclure que c'est en fait parce que ces sciences prétendent aujourd'hui occuper, sans partage, ce licu de connaissance privilégié qu' elles refoulent tout questionnement éthique, qu'cllcs se soustraicnt à lcur unique légitimité : alimenter un débat politique constant de la société avec elle-même.

La seconde stratégic suit un raisonnement en fait assez proche. Elle pose que l'exi- gence normative n'cst pas sculcment corrélative de ce questionnement général où s'originent les sciences sociales. Elle affirme, dans le prolongement du tournant langagier qui a marqué la sociologic compréhensive depuis déjà une trentaine d'annćes (3)-, que cette normativité est inscrite dans la nature même des concepts propres aux sciences sociales. La prétention de ces sciences à constitucr un métalangage, occupant ce licu surplombant le langage ordinaire, s'effondre dès lors que l'on souligne que tout cadre conceptuel secrète inexorablement une conception du bien, un ensemble d'ćvaluations normatives, logées dans le langage même de la science qui, pour cctte raison, ne saurait être radicalement dissocié du langage ordinaire. Cette nature intrinsèquement normative de nombre de concepts sociaux (ct donc sociologiques) a pour conséquence leur caractère "essenticllement contestable". Ainsi, si toute analyse en science sociale ne peut faire usage que des concepts constitutifs des pratiques qu'elle étudie (absence de méta-langage) et si ccux-ci font l'objet d'interprétations contestées (contestabilité essentielle), alors l'analyste ne fait rien d'autre que de choisir parmi ces interprétations et donc ne saurait échapper à cette contestabilité ct écarter les exigences du débat, de l'argumentation, tant scientifique, ćthique que 
politique.

On le voit, cet ouvrage a le mérite, en pointant ces deux tentations qui ont stérilisé voire assombri le projet moderne des sciences sociales : celle de la connaissance pour la connaissance et de la connaissance pour la puissance, de placer les sciences sociales face à lcur indétermination constitutive. Le constat de cette indétermination conduit moins à une quelconque désillusion qu'à une invitation à assumer cette normativité et cette contestabilité intrinsèques de l'analyse sociale. Et le lieu de cette responsabilité, éthique et politique, s'il ne saurait plus être celui du Savoir et du Pouvoir, n'est autre que cet espace discursif, espace de débat où ces disciplines, à travers lesquelles nos sociétés tentent de s'appréhender, acceptcront de s'abandonner à ce questionnement interminable sur leur nature et leur finalité.

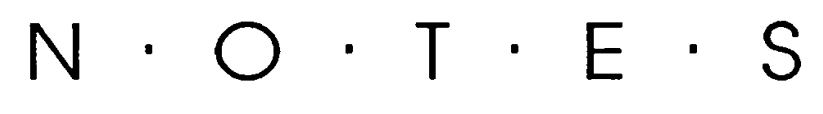

1. Il s'agit de la grande enquête menée par R.N Bellah et ses collaboratcurs, Ilabits of the Heart. Individualism and Commitment in American Life, Harper and Row, 1986 dont l'originalité de la démarche est explicitée en fin de parcours dans une appendice stimulante intitulée "Social Science and Public Philosophy" (p.297-307).

2. Telle est, trop rapidement évoquée, la thèse proposée par Alain CAILLE dans son ouvrage La Démission des Clercs. La crise des sciences sociales et l'oubli du politique, La Découverte, 1993.

3. Ce questionnement, influencé de Wittgenstein et de la tradition analytique anglosaxonne, s'origine principalement dans l'ouvrage désormais classique de Peter WINCH, The Idea of a Social Science and its relation to philosophy, Routledge and Keagan Paul, 1958.

- Il a suscité depuis lors, directement ou indirectement, nombre de débats dont les protagonistes sont notamment C. Taylor, A.Mac Intyre, A.R Louch. Voir par exemple, C.Taylor, "Interpretation and the Science of man" et "Neutrality in Political Science", in Philosophical Papers II, Cambridge University Press, 1985 ; A.Mac Intyre, "The essential 
contestability of some social concepts", in Ethics, vol.84, n¹, 1973 ; A.R Louch, Explanation and II uman Action, University of California Press, 1969. 